Revue d'histoire de l'Amérique française

REVUE D.HISTOIRE DE L'AMÉRIQUE FRANÇAISE

\title{
Les Acadiens dans une seconde patrie : La Louisiane
}

\section{Jeanne Grégoire}

Volume 15, numéro 4, mars 1962

URI : https://id.erudit.org/iderudit/302156ar

DOI : https://doi.org/10.7202/302156ar

Aller au sommaire du numéro

Éditeur(s)

Institut d'histoire de l'Amérique française

ISSN

0035-2357 (imprimé)

1492-1383 (numérique)

Découvrir la revue

Citer cet article

Grégoire, J. (1962). Les Acadiens dans une seconde patrie : La Louisiane. Revue d'histoire de l'Amérique française, 15(4), 572-593.

https://doi.org/10.7202/302156ar d'utilisation que vous pouvez consulter en ligne.

https://apropos.erudit.org/fr/usagers/politique-dutilisation/ 


\section{LES ACADIENS DANS UNE SECONDE PATRIE : LA LOUISIANE}

Les manifestations organisées en 1955, à l'occasion du bicentenaire de la déportation des Acadiens, ont donné lieu à des pèlerinages qui permirent de nouveaux contacts entre les familles de l'ancienne Acadie et celles de Nouvelles Acadies nées au Québec aussi bien qu'aux États-Unis d'Amérique. L'un de ces nouveaux centres, objet de manifestations qu'on pourrait qualifier de spectaculaires, fut celui qui prit naissance au Poste des Atakapas, sur le bayou Tèche, en Louisiane, avec l'arrivée, en 1765, d'un groupe d'exilés désemparés, meurtris par la souffrance et la faim qui trouvèrent dans cette oasis de fraîcheur et de verdure, le «havre de Grâce » désiré, après leur séjour au Maryland. Ils en firent leur patrie d'adoption et entreprirent immédiatement l'érection d'une chapelle dont une partie servit, en 1832, à la construction de l'église actuelle. En briques faites à la main, surmontée de deux clochers, cette église domine la place, devenue un lieu de pèlerinage ethnique annuel pour tous les Acadiens de la région et un lieu de rassemblement lors du passage des pèlerins venus des Maritimes et du Québec en mars 1955. Le parc, les monuments qui s'y trouvent, sans en être une réplique, sont un rappel de ceux qui ont été élevés à SaintCharles-des-Mines, en Acadie, mieux connu sous le nom de Grand-Pré.

L'église en forme de croix, la croix dont s'orne généreusement chaque fenêtre rappellent la haute croix noire élevée sur la plage d'embarquement à Gaspareau d'où partit un peuple affolé, en 1755, vers une destination inconnue. Un peu en retrait, en arrière de l'église, s'élève un chêne gigantesque au riche feuillage toujours vert, qu'on nomme le « chêne d'Évangéline » et qu'on dit «être du nombre des arbres fameux», et membre de «The Live Oak Association»; il résume la lignée de vieux saules 
formant une sorte d'arrière-garde au parc de Grand-Pré. Tout près, s'élève le monument commémoratif d'Évangéline, sur le terrain du «vieux cimetière de Saint-Martin, à la mémoire des Acadiens exilés qui arrivèrent en ce lieu en 1765 », comme l'indique l'inscription gravée sur un socle solide portant bien assise, une Évangéline pensive, nostalgique, le regard fixé sur un horizon de mystère, tandis que le monument de Grand-Pré montre une Évangéline debout, la tête tournée, pleurant le pays qu'elle vient de quitter. A une distance d'environ un mille, s'étend le parc commémoratif Longfellow où, d'après la légende, se trouve la maison de Gabriel Lajeunesse, convertie en un musée historique, tout comme l'est maintenant l'église de GrandPré.

Telle se trouve aujourd'hui la ville édifiée avec amour et ferveur par un noyau détaché, par la force des circonstances, de l'ancienne Acadie et où, dans ce coin de la Louisiane, un sol riche et plantureux pourvoit à leur subsistance, une Terre Promise quoi, qui répondant à leurs efforts, produit cent pour un puisque ce sol fertile peut donner annuellement jusqu'à quatre récoltes. Outre l'exploitation des ressources naturelles: sel, huile, bois, on y cultive principalement la canne à sucre, le riz et le coton, puis on utilise, à divers usages, une mousse espagnole apportée par le vent qui flotte aux branches des chênes et des cyprès formant une abondance de draperies funèbres permannentes à l'ombre desquelles les exilés gagnèrent leur pitance par le travail de leurs bras. Ils puisaient à même la forêt, le bois qui leur servait à la construction de leur masure, au chauffage de leur habitation et à la fabrication de leurs embarcations. Ces grands arbres qu'ils abattaient et sectionnaient étaient transportés au moyen de pirogues qu'ils glissaient doucement dans les méandres des bayous, sur les lacs nombreux et dans les eaux du grand fleuve. Ces embarcations leur étaient d'un grand service pour «faire la traversée d'un bord de rivière à l'autre, ou pour y charroyer les provisions des champs, nous disent les Relations des Jésuites (1672-74: 96); elles étaient très populaires et indispensables; sur le Mississipi, les pirogues sont en si grand nombre qu'en une seule bourgade on en vit jusqu'à 280 
ensemble. Il y en a cependant toujours quantité aux environs des villages ».

\section{HISTORIQUE}

Le Poste des Atakapas, le plus ancien centre acadien de l'État de la Louisiane, est en fait le cœur du pays d'Évangéline. Établi en 1756, il paraît avoir eu comme premier colon, Gabriel Fuselier de la Claire, qui, en 1760, acheta de Rinemo, chef de cette tribu des Atakapas, des villages situés à l'ouest de ce qui est aujourd'hui Saint-Martinville. Après leur licenciement de l'armée, plusieurs officiers français s'y établirent en même temps que des familles espagnoles. Deux ou trois ans plus tard, un ex-capitaine de l'armée française, le Marquis de Vaugine exploite, non loin de là, une plantation d'indigo.

Le Gouvernement espagnol établit, en 1797, des lois régissant l'organisation de la colonie, lois par lesquelles on favorise de préférence l'établissement de colons catholiques; les protestants y sont acceptés mais on ne tolère pas la prédication en faveur de leur religion. Des aristocrates fuyant la Révolution vinrent de France s'y établir et tenter de continuer leur train de vie social et culturel; le Poste des Atakapas devint alors «le Petit Paris » de la Louisiane. Cependant le changement de domination du Gouvernement de France à celui de l'Espagne, mit un terme aux extravagances de ces aristocrates.

\section{ARCHIVES}

Quels sont en fait les premiers Acadiens qui peuplèrent cette nouvelle contrée? Des perquisitions dans les Archives judiciaires et paroissiales révèlent ces noms qui sans elles resteraient incertains. Les premiers documents d'archives du Palais de Justice remontent à quelque cinq ans après l'arrivée des Acadiens, c'est-à-dire à 1770, et sont les plus anciens de la Louisiane. Les autres archives susceptibles de nous éclairer, de nous fournir des précisions sur l'effectif du groupe acadien dans cette région, sont sans contredit, les registres de baptêmes, mariages et sépultures qu'on est convenu d'appeler les registres 
paroissiaux, tenus tant bien que mal par les missionnaires du temps. Ceux-ci ont exercé leur ministère dès l'arrivée des exilés.

Nous devons à M. Miguel Berud Dassiese, curé de SaintMartin des Atakapas, d'avoir collationné, en 1796, tous les actes qu'il a pu trouver « dans de vieux cahiers, sur des feuilles volantes ou des coupons aux archives de cette paroisse depuis le premier prêtre qui y a exercé des fonctions en 1756 jusqu'en 1782 où les registres ont commencé à avoir plus d'ordre et d'exactitude », selon sa propre expression. M. Dassiese y a inséré des notes explicatives à plusieurs endroits: il mentionne que les actes de ces temps qui ne s'y trouveront pas pourront se retrouver à la Pointe Coupée, ${ }^{1}$ aux Opelousas, aux Natchitoches, ${ }^{2}$ à la Fourche, ${ }^{3}$ ou enfin à la ville (la Nouvelle-Orléans).

Ce premier registre de Saint-Martinville contient d'abord les actes d'un «vieux et petit registre » colligé par les soins du Père Junée, capucin, curé de la Pointe Coupée, et envoyé anciennement par lui dans cette paroisse. Le seul acte inscrit en 1756, est celui du baptême d'une sauvagesse de la nation Cancy, âgée d'environ 7 ans, et nous sautons à 1762, alors que le Père Valentin, cap., curé de la paroisse Saint-François des Natchi-

${ }^{1}$ La Pointe Coupée qui s'étend sur une superficie de 564 milles carrés, fut érigée en paroisse à la même date que celles de St-Landry des Opelousas, de Saint-Gabriel et des Natchitoches, le 31 mars 1807. Elle fut ainsi nommée par d'Iberville qui en fit l'exploration en 1699, et y ouvrit une artère lui donnant la forme d'une pointe, pour faire venir l'eau du Mississipi.

Des trappeurs canadiens y vinrent aussi tôt qu'en 1708; elle reçut aussi des déserteurs de la colonie française de Biloxi. Bienville y établit un poste et distribua des terres aux colons.

${ }^{2}$ Les Natchitoches se composent de six petites îles sur la rivière Rouge; elles sont recouvertes d'une forêt de pins et s'étendent sur une superficie de 1297 milles carrés. Elles furent colonisées par les Français, et en partie par les Espagnols, en 1717. La paroisse dite des Natchitoches fut érigée le 31 mars 1807; elle est placée au deuxième rang dans l'Etat de la Louisiane pour son étendue en culture. Les premiers registres des baptêmes, mariages et sépultures de la paroisse des Natchitoches dont je possède le microfilm que j'ai lu, ne contiennẹt aucun nom acadien pour la période allant de la fondation de la paroisse jusqu'à 1795 inclusivement.

${ }^{3}$ Lafourche, érigée en paroisse le 10 avril 1805, est l'une des plus grandes de cette région; elle tire son nom du Bayou Lafourche qui a une configuration de fourchette du Mississipi. Ce bayou possède aujourd'hui, sur une longueur d'environ cent milles sur ses deux rives, une des plus denses populations rurales des Etats-Unis; le siège de Lafourche est Thibodeaux. Sa superficie est de 1157 milles carrés. 
toches exerce les fonctions en passant aux Atakapas, et y baptise, le 18 février, trois enfants grifs. Après cette date, on ne retrouve plus d'actes qu'en 1765, alors que les Acadiens y arrivèrent accompagnés du Père Jean-François, capucin français, qui remplit les fonctions de pasteur durant un an, dans ce poste qu'on nommait allors Nouvelle Acadie. M. Dassiese ajoute qu'au rapport des anciens, il a baptisé et enseveli beaucoup de monde mais que trente-six actes seulement de sépultures ont été retrouvés, et il fait remarquer qu'au commencement et à la fin du registre, plusieurs feuillets ont été détachés à l'aide d'un canif; peut-être, ajoute-t-il, « cette mission n'ayant point de consistance solide, la prudence a exigé d'enlever les extraits pour les emporter et les transcrire sur les livres de la paroisse, à la ville, ou dans ceux des paroisses ci-dessus mentionnées ».

Le registre contient pour cette seule année 1765, cinq baptêmes et trente-six sépultures. En l'an 1766, un seul acte de baptême y est inscrit, et nous arrivons à l'année 1771, marquée par treize actes de baptême et trois actes de mariage, aucune sépulture.

Les actes de l'année 1771 ne portent que la date de naissance de l'enfant, la date de baptême n'y apparaît pas. Cependant, à la seule date du 25 avril, sont inscrits deux actes de mariage et un acte de baptême.

Pour l'année 1772 , le P. Junée, capucin, curé de la Pointe Coupée, étant à la cure vacante des Atakapas, supplée la cérémonie du baptême, le 13 juillet, à une fille, née le 8 février dernier, et deux jours plus tard, il baptise un garçon né le $\mathbf{1 5}$ décembre précédent et neuf autres enfants nés au cours des mois précédents, puis suivent 5 actes de mariage sans date précise; le 17 juillet il baptise 4 enfants nés au cours des douze mois écoulés.

L'année 1773 compte plusieurs actes de baptême sans date précise et quelques actes de mariage; le registre se ferme avec une attestation du Rév. Père Junée, Capucin, curé de la Pointe Coupée, sur la vérité des actes ci-dessus : 
Je soussigné prêtre Capucin Missionnaire, curé de la Pointe Coupée, certifie les actes de Baptême et de mariage contenus en ce cahier, être véritables et conformes à leurs originaux ayant été tirés mot-àmot des feuilles volantes collées dans des Registres de la Paroisse de la Pointe Coupée des Registres mêmes et des différents cahiers dans lesquels j'avais inscrit les actes de Baptême et mariage que j'ay faits dans les diférens voyages que j'ay fais au Atakapas lors de la vacance de la Cure, et y étant appellé par les habitans, l'on doit ajouter foy aux dits actes, tant en justice que dehors, en témoin de quoy j'ay fait et signé le présent pour valoir et servir ce que de raison partout du besoin sera à la Pointe Coupée ce quinze janvier mil sept cent soixante et quatorze et est signé

\section{F. Junée Capucin}

NOTA: Les extraits suivants sont tirés de feuilles volantes sans date si ce n'est à côté en marge où l'on trouve des dates qui indiquent sans doute le jour de la naissance de ceux qui ont été baptisés, ces feuilles sont en très grand désordre, il y a apparence qu'elles ont été envoyées par le même Père Junée, car à la fin de l'année 1774, on trouve sa seule signature; cependant, on trouve encore un seul acte sur une demi-feuille volante d'un baptême fait dans cette paroisse par le Père Louis-Marie, curé des Opelousas. Il paraît que le Père Junée a fait cette mission en novembre 1774.

On ne trouve point d'actes pour l'année 1775; les actes inscrits en l'année 1776, sont tous datés du 5 mai et sont écrits par la même main que ceux de l'année 1774, mais ne portent aucune signature.

En l'année 1777, existent, dans le registre de papier ordinaire, deux feuilles d'écriture différente ne portant aucune signature mais qui sont sûrement du Père Louis-Marie, cap. Le nom d'Achille Bérard se trouve à la fin de l'année 1779, sur une feuille écrite en espagnol; c'est une enquête faite pour lui le premier février. Suivent des extraits d'actes trouvés sur des coupons et qui ont été faits par le Père Bernard de Deva, capucin, 
curé de cette paroisse dans les années 1788-89-90 et 1791. C'est son écriture, note $\mathrm{M}$. Dassiese, mais ils ne portent aucune signature ni de date pour l'année. Une autre note mentionne qu'il existe encore des extraits pris sur des coupons ou feuilles volantes laissés par George Murphy, «mon prédécesseur immédiat vers le temps qu'il devait quitter le poste; ils sont au nombre de trois et comme je connais les familles, je les mets un peu plus étendus qu'il ne les a laissés ». L'année 1796 se termine par une attestation signée le 6 septembre 1796, par Migl Bernd Barrière, curé des Atakapas, certifiant avoir fait ce travail avec toute l'exactitude possible.

Les nombreuses notes écrites par MM. Dassiese et Barrière indiquent clairement la teneur de ce premier registre de SaintMartin des Atakapas dont le résumé donne les statistiques suivantes pour les années courant de 1765 à 1794 inc. : baptêmes 166 - mariages 19 - sépultures 38 dont 36 au cours de la seule année 1765 .

Cette nouvelle Acadie au Poste des Atakapas fut érigée en paroisse le 31 mars 1807, et reconnue officiellement sous le vocable de Saint-Martin, le 17 avril 1871. Par les mentions écrites dans certains actes de sépulture assez détaillés, on découvre que les Acadiens étaient dispersés par groupes en plusieurs endroits que l'on distinguait par le ler camp, le camp d'en haut, le 1er ou dernier camp d'en bas, le camp Beausoleil; plusieurs camps formaient une paroisse tandis qu'à Belle Isle en Mer, chaque groupe formait un village et plusieurs villages constituaient une seule paroisse.

\section{LA PAROISSE SAINT-LANDRY DES OPELOUSAS}

Les deux districts des Atakapas (Saint-Martinville) et des Opelousas (Saint-Landry), séparés par un petit bayou, le Tèche, ont une étendue de 25 lieues de longueur et 5 lieues de largeur; ils sont entourés de forêts sillonnées de plusieurs bayous.

La paroisse portant le nom civil des Opelousas s'étend aujourd'hui sur une superficie de 930 milles carrés et fut colo- 
nisée par des Français et des Anglais qui y ont été attirés par la fertilité et la richesse du sol. Elle fut érigée canoniquement le 31 mars 1807, à la même date que trois des autres paroisses environnantes, et fut reconnue sous le vocable de Saint-Landry; en outre de rappeler la mémoire d'un saint qui vécut au sixième siècle, il convenait de lui donner ce nom si répandu (par milliers) dans l'état de la Louisiane.

Le registre des baptêmes qui s'ouvre le 19 mai 1776, se ferme en avril 1785, avec un total de 113 actes; celui des mariages commence le 15 janvier 1784 pour se terminer le 24 novembre 1795, avec un total de 169 actes dont 16 sont illisibles; celui des sépultures débute le 27 octobre 1779 et couvre la période écoulée jusqu'au 10 mai 1806, pour former un total de 496 actes. A partir de 1779, les actes sont signés par Joseph de Arazenoff, curé, jusqu'au mois de juin 1789 alors qu'apparaît le nom de Fr Pedro de Tamora, jusqu'à la venue, en juillet 1801, de M. Louis Buhot. Il ne se trouve qu'une seule sépulture en 1784 et trois en 1785; on fait toujours mention dans ces actes de la paroisse Saint-Landry, Ibérie, diocèse de la Havane.

Le livre des mariages entièrement rédigé en langue espagnole (à l'exception de quelques rares actes en langue française) jusqu'au 25 décembre 1803, l'est en langue française jusqu'au 10 mai 1806; il s'ouvre sur une attestation datée du ler janvier 1908, de M. Francis Léon Ganler, curé, qui dit le nombre d'actes de mariages contenus dans ce registre, plus quatre entrées de baptêmes par le Père Joseph de Arazenoff qu'il a copiés du registre original des baptêmes et auxquels se joint un petit registre contenant vingt-huit actes complets et deux incomplets, plus quelques feuilles du registre original commencé en 1777 par le Père Louis, cap.

Une note apparaît en tête de la première page du registre des sépultures pour l'année 1798, disant que « l'église actuelle fut transférée du Bayou sur le local appelé la Pointe à $\mathrm{M}$. Tesson, que M. Michel Prud'homme, natif des environs et près de Strasbourg, en France, donna gratuitement trois arpents de terre de face sur quarante de profondeur, et que ce M. Tesson, natif de la Xaintonge en donna un ». 
A la fin de 1791 existe une liste de sept noms de personnes sans date de sépulture; on y mentionne parfois que le défunt a été enterré par charité. Il est à noter que malgré la vie dure et rude de colons-défricheurs, il s'est trouvé un centenaire parmi les Acadiens, Michel Doucet, enterré le 13 octobre 1805, âgé de 105 ans. Entre les dates du 14 mars et du 30 mai 1804, apparaît une nouvelle liste de huit noms de personnes qui ont été enterrées par le sacristain en l'absence du curé de la paroisse. Le registre des sépultures pour cette paroisse des Opelousas, se clôt par le certificat suivant:

Je certifie que le R.P. Fr Pedro de Zamora ma rendu tous les comptes des enterrements cy-dessus qui sont dus à la Fabrique depuis le 11 août 1791 jusqu'au 31 décembre 1795 en ayant plusieurs personnes qui n'ont pas encore payé comme il comte par le compte détaillé que ledit R.P. m'a livré, et dont je l'enregisterai dans le livre de fabrique;

Opelousas 31 Enero 1796

Jean Gradenigo
Mayordomo

Je dois avouer que le défrichement des actes de ces registres sur microfilm a été plutôt pénible, étant donné leur mauvais état; certains actes sont en partie effacés tandis que d'autres ayant absorbé l'encre qui a pénétré au point de traverser toute l'épaisseur du papier sont tout à fait illisibles; certains actes ont été insérés tête-bêche tandis que des pages entières ont été placées aussi tête-bêche. Il faut ajouter à cela la rédaction en langue espagnole de la presque totalité des mariages et des sépultures. Il ne faut pas s'en étonner puisque la Louisiane a connu quarante années de domination espagnole, que dix drapeaux y ont flotté jusqu'à sa cession aux Etats-Unis en 1812.

\section{LA PAROISSE SAINT-GABRIEL D'IBERVILLE}

La paroisse Saint-Gabriel d'Iberville, Côte de Manchac, s'étend sur une superficie de six cent onze mille carrés; érigée le 31 mars 1807, elle fut ainsi nommée en mémoire de l'explo- 
rateur d'Iberville, frère de bienville qui fit son premier voyage au Mississipi en 1699. Durant cette même année, un missionnaire jésuite, le Père $\mathrm{Du} \mathrm{Ru}, \mathrm{y}$ établit une mission en vue de la conversion des Indiens Bayougoulas. En 1769, la population ne comptait encore que trois cent soixante-seize personnes; la plupart étant des Acadiens qui formaient la plus importante colonie.

\title{
Registres paroissiaux
}

On trouve en première page du registre de cette paroisse que j'ai consulté aux Archives d'Ottawa l'attestation suivante:

Je, soussigné, A.-M. Barbier, Recteur de l'église StGabriel, en Louisiane, certifie que la transcription et traduction des registres de mariages (suivants de St-Gabriel, de 1773 à 1859 inclus) est une vraie copie des registres de la paroisse de Saint-Gabriel de la Louisiane, Etats-Unis de l'Amérique du Nord.

En foi de quoi je signe et appose le sceau de ladite église.

\author{
AM Barbier \\ Curé de St Gabriel.
}

Le registre s'ouvre en janvier 1773 avec les actes de baptêmes; il y en eut 19 au cours de l'année. Les cinquante-trois premières pages comprenant 26 actes du registre des mariages contiennent des noms espagnols, polonais, irlandais, allemands, cubains; tous les actes sont rédigés en langue espagnole. Pour les années courant de 1773 à 1795, l'on compte quatre-vingt-six mariages dont au moins l'un des conjoints porte un nom acadien, mais la plupart sont des mariages entre acadiens. Dans les autres actes, les noms de lieux d'origine mentionnés sont variés à l'infini. Nous n'avons fait le relevé que des actes portant des noms acadiens.

\section{LA PAROISSE SAINT-LOUIS DE LA NOUVELLE-ORLEAANS}

Le premier mariage inscrit au registre de la cathédrale Saint-Louis, Nouvelle-Orléans, date du 1er juillet 1720. A partir 
de janvier 1765, apparaissent les noms acadiens, de gens qu'on dit natifs de Chipoudy, de l'Ile Saint-Jean, de Beaubassin, d'Annapolis Royal, de Pointe Beauséjour, puis de Québec et Montréal.

Les noms acadiens aux registres de la Louisiane ont considérablement augmenté à partir de 1785 , alors que six frégates et un bateau ramenèrent de France bon nombre d'Acadiens désireux de venir rejoindre leurs compatriotes. L'ouvrage de Winzerling, intitulé Acadian Odysse donne des listes de noms de personnes avec mention des lieux où on les a conduits en Louisiane.

Voilà résumé, l'état des registres et de leur contenu de trois paroisses de la Louisiane que formèrent les Acadiens de la Déportation: Saint-Martinville, Saint-Gabriel et Saint-Landry où furent enregistrés les événements habituels, ordinaires qui surviennent au cours du développement normal d'une population; il ne m'a pas été donné de pouvoir faire le relevé de la paroisse Saint-Louis ni des trois autres mentionnées au registre de SaintMartinville.

La Louisiane fut pour ces exilés désemparés, le havre de sécurité rêvé après leur départ de leur patrie d'origine; c'est là que, fourbus, torturés par la faim et la fatigue, pleurant leurs belles et riches fermes d'Acadie, leurs riches ports de pêche, les attelages de boeufs, les exilés se bâtirent de nouvelles demeures, travaillant avec ardeur et satisfaction, un sol riche et fertile qui répondait généreusement à leur labeur quotidien. Ils s'adaptèrent et s'attachèrent à leur patrie d'exil qui devint pour eux un paradis sur terre. Et c'est ainsi qu'on vit naître une Nouvelle Acadie sur les bords du grand fleuve Mississipi.

\section{PAROISSE SAINT-MARTIN DES ATAKAPAS, LOUISIANE}

Le premier acte inscrit au registre de Saint-Martinville porte la date du 5 juin 1756, et représente le certificat de baptême d'une petite sauvagesse de la nation Cancy, âgée d'environ sept ans, à qui on a donné le nom de Thérèse Labombe, le parrain et la marraine furent des nègres libres; on mentionne que la mar- 
raine est une sauvagesse, esclave de M. Masse, aux Atakapas. L'acte est signé : Didier, prêtre.

Le relevé suivant que nous reproduisons en résumé dans l'ordre alphabétique, classé par groupes des baptêmes, mariages et sépultures, a été fait d'après un vieux registre, 1ère partie, en possession de M. le chanoine Peters, curé de Saint-Martinville, dont copie a été tirée en mai 1922, par Mlle Olga Bienvenue sous la direction de M. Dan Woorhies, avocat à Saint-Martinville.

\section{BAPTÊMES 4a}

Babinaud, Anne, 1774, 10 oct. 1774, Charles, Anne Guilbaut.

Babinaud, David, 15 juil. 1772, 25 av. 1771, Charles, Anne Guilbaut.

Babinaud, Julien, 21 sept. 1794, janv. 1792, Joseph, Félicité Cormier.

Bara, Alexandre, 29 avril 1773, 25 janv. 1773, Vincent, M.-Françoise Labbé.

Bara, Hyppolite, 24 nov. 1776, 15 mars 1776, Vincent, M-Françoise Labbé.

Bara, Jean-Baptiste, 15 juil. 1772, 20 fév. 1772, Vincent, M-Françoise Labbé.

Bara, Valery, 18 juil. 1778, 27 sept. 1777, Vincent, M-Françoise Labbé.

Bérard, Adélaide, 2 janv. 1771, 11 déc. 1770, Jean, Anne Broussard.

Bérard, Alexandre, 24 nov. 1776, 3 mars 1775, Jean, Anne Broussard.

Bérard, Camille, 19 juil. 1778, 19 sept. 1777, Jean-Bte, Anne Broussard.

Bérard, Jean-Baptiste, 1 mai 1773, 15 fév. 1773, Jean-Bte, Anne Broussard.

Bergeron, Marie-Anne, 4 août 1765, 31 mai 1765, Jean-Bte, Catherine Guessi.

Bernard, Marie, 4 déc. 1774, — Michel, Marie Guilbaut.

Bernard, Marie-Anne, 2 janv. 1771, 7 sept. 1770, Michel, Marie Guilbaut.

Bigeau, Auguste, 17 juil. 1778, 23 mars 1778, Augustin, Anne Landry.

Bonin, Jean-Louis, 5 mai 1776, — Jean-Louis, Marguerite Prince.

${ }^{4 a}$ Cette liste de baptêmes est donnée dans l'ordre suivant: nom, date de baptême, date de naissance, père, mère. 
Bonin, Louise, ${ }^{4 b} 27$ août 1777, 28 mars 1777, Jean-Louis, Marguerite Prince.

Bonin, Suzanne, 25 avril 1773, 28 mars 1773, Jean-Louis, Marguerite Prince.

Borda, Modeste, 17 juil. 1772, 24 janv. 1772, Antoine, Marguerite Martin.

Borda, Scholastique, 2 janv. 1771, 18 fév. dernier, Antoine, ${ }^{5}$ Marguerite Martin.

Borel, Victoire, 26 avril 1773, 16 mars 1773, Pierre, Catherine Toupard.

Brault, Félicité, 5 mai 1776, -, Firmin, Marguerite Brau.

Broussard, Adélaide, 1774, 26 juin 1774, Sylvain, Félicité Guilbaut.

Broussard, Alexandre, 24 août 1777, 15 déc. 1777, Simon, Marguerite Blanchard.

Broussard, Anaclet, 2 janv. 1771, 7 oct. 1770, Sylvain, Félicité Guilbaut.

Broussard, Apollonie, 25 avril 1773, 5 fév. 1773, Claude, Louise Hébert.

Broussard, Apollonie, 5 mai 1776, _, Sylvain, Félicité Guilbaut.

Broussard, Apollonie, 24 nov. 1776, 25 nov. 1775, Augustin, Anne Landry.

Broussard, Baptiste, 1774, 19 oct. 1774, Claude, Louise Hébert.

Broussard, Constance, 10 janv. 1779, 9 oct. 1778, Augustin, Anne Landry.

Broussard, Edouard, 12 juil. 1778, 15 oct. 1777, Amand, Anne Benoit.

Broussard, Félicité, 24 oct. 1777, 12 juil. 1777, Sylvain, Félicité Guilbaut.

Broussard, François, 10 janv. 1779, 2 janv. 1779, François, Pélagie Landry.

Broussard, Frs-Alexandre, ${ }^{4 b} 26$ août 1777, 20 mars 1777, Joseph, Marguerite Savoye.

Broussard, Hubert, 29 avril 1773, 3 août 1772, Sylvain, Félicité Guilbaut.

Broussard, Jean, 5 mai 1776, - François, Pélagie Landry.

Broussard, Josaphat, 17 juil. 1772, 29 nov. 1771, Amand, Hélène Landry.

Broussard, Joseph, 24 août 1777, 15 juin 1777, Pierre, Marie Melançon.

Broussard, Joseph, 26 août 1777, 16 mai 1777, François, Pélagie Landry.

4b Baptisé à la paroisse Saint-Bernard.

5 Antoine est dit « chirurgien du poste des Atakapas 》. 
Broussard, Joseph, ${ }^{\text {bb }} 26$ août 1777, 22 mars 1777, Joseph, Anne Brault.

Broussard, Joseph, 24 nov. 1776, 15 mars 1774, Joseph, Marguerite Savoye.

Broussard, Louis, ${ }^{4 b} 27$ août 1777, 25 août 1777, Claude, Louise Hébert.

Broussard, Magdeleine, 18 juil. 1772, 26 mars 1772, Joseph, Marguerite Savoye.

Broussard, M-Magdeleine, 24 nov. 1776, 20 fév. 1776, Junée, MMagdeleine Landry.

Broussard, Marguerite, 29 avril 1773, 11 déc. 1772, Simon, Marguerite Blanchard.

Broussard, Marguerite, 3 janv. 1779, 27 oct. 1778, Joseph, Anne Brault.

Broussard, Odilon, 2 janv. 1771, _, François, Pélagie Landry.

Broussard, Perpétue, 2 janv. 1771, 14 avril 1770, Jean-Baptiste, Anne Brun.

Broussard, Simon, 2 janv. 1771, 4 mars 1770, Simon, Marguerite Blanchard.

Broussard, Théophile, 25 avril 1773, 5 mars 1772, François, Pélagie Landry.

Broussard, Valéri, 27 août 1777, 15 mai 1776, Claude, Louise Hébert.

Carlin, Alexis, 15 juillet 1778, 5 déc. 1773, Joseph, Françoise Lange.

Carlin, Eugène, 15 juill. 1778, mars 1775, Joseph, Françoise Lange.

de Clouet, J-Augustin, 19 juill. 1778, 9 janv. 1778, Alexandre, ${ }^{6}$ Louise Favreau.

de Clouet, M-Louise Hyacinthe, 25 août 1777, 8 avril 1776, M. le Chevalier, ${ }^{7}$ Anne-Louise Favreau.

Decoux, Hilaire, 19 juill. 1778, 12 mai 1778, Joseph, Charlotte Labbé.

Decoux, M-Marguerite, 29 avril 1773, 10 avril 1773, Joseph, Charlotte-Julie Labbé.

De Cuir, Godefroy, 18 juill. 1778, 12 mai 1777, François, Marie Labbé.

De Roan, Joseph-Marie, 24 nov. 1776, 19 nov. 1776, Joseph, Geneviève Hébert.

Doucet, Joseph, 5 mai 1776, - Joseph, Anne Landry.

Doucet, Marie, 1774, 9 oct. 1773, Hilaire, Anne Landry.

\footnotetext{
${ }^{6}$ Alexandre de Clouet est dit « chevalier $\$$.

${ }^{7} \mathrm{M}$. le Chevalier de Clouet, \& capitaine d'infanterie et Commandant pour le Roy $\gg$.
} 
Ducrest, Julie, 1 mai 1773, 27 fév. 1773, Louis-Armand, JeanneCatherine Wilse.

Ducrest, Louise, ${ }^{4 \mathrm{~b}} 25$ août 1777, 19 juin 1777, Louis, ${ }^{8}$ Anne-Catherine Wilse.

Dugas, Augustin, 2 janv. 1771, 20 fév. 1770, Jean, Marguerite Dupuis.

Dugas, Félicité, 1774, 4 juill. 1774, Jean, Marguerite Dupuis.

Dugas, Françoise, 25 août 1777, 14 juin 1777, Pierre, Anne Thibodeau.

Dugas, Jean, 24 août 1777, 10 juill. 1777, Jean, Marguerite Dupuis.

Dugas, Julie, 17 juill. 1772, 16 avril 1772, Jean, Marguerite Dupuis.

Dugas, Louis, 24 nov. 1776, 18 nov. 1776, Charles, Marguerite Broussard.

Dugas, M-Magdeleine, 29 avril 1773, 22 avril 1773, Charles, Marguerite Broussard.

Dugas, Pierre, 24 août 1777, 6 mars 1777, Pierre, Anne Thibodeau.

Dugas, Silésie, 1774, 26 oct. 1774, Pierre, Anne Thibodeau.

Duser, Anselme, 19 juil. 1778, 3 oct. 1777, Joseph, Anne Landry.

Fostin, Jacques, 10 janv. 1779, 18 déc. 1778, Jacques, Françoise Trahan.

Fostin, Marguerite, 1774, 29 mai 1773, Jacques, Françoise Trahan.

Fostin, M-Angélique, 5 mai 1776, _, Jacques, Françoise Trahan. Fuselier, Hélène, 13 juill. 1772, 8 fév. 1772, Gabriel, ${ }^{9}$ Hélène Soileau.

Guilbaut, Anastasie, 1774, 2 juin 1774, François, Magdeleine Broussard.

Guilbaut, Armand, _, 2 mai 1778, Charles, Marguerite Bourque.

Guilbaut, Emile, 1774, 20 déc. 1773, Charles, Anne Trahan.

Guilbaut, François-Louis, 5 mai 1776, —, François, Madelon Broussard.

Guilbaut, Jean-Charles, 15 juill. 1772, 15 déc. 1771, Charles, Anne Trahan.

Guilbaut, Joseph, 24 août 1777, 15 avril 1777, François, Magdeleine Broussard.

Hébert, Adélaïde, 1774, 2 mai 1774, Joseph Pépin, Magdeleine Trahan. kapas.

${ }^{8}$ Louis Ducrest, officier, major de la Compagnie de milice des Ata-

${ }^{9}$ Gabriel Fuselier de la Claire, commandant et juge civil aux Opelousas et Atakapas. 
Hébert, Agricole, 1 déc. 1776, 8 oct. 1776, Joseph, Magdeleine Trahan.

Hébert, Céleste, $^{4 b} 25$ août 1777, 12 fév. 1777, Jean, Théotiste Hébert.

Hébert, Constance, 18 août 1778, le même jour, Joseph, Françoise Hébert.

Hébert, Joseph, 15 juill. 1772, 25 mars 1772, Joseph, Françoise Hébert.

Hébert, Marie, 1774, 1774, Charles, Magdeleine M. Cadet.

Hébert, Marie, 18 juill. 1772, 5 nov. 1771, Jean-Baptiste, Théotiste Hébert.

Hébert, Scholastique, 5 mai 1776, - , Jean-Baptiste, Théotiste Hébert.

Hébert, Scholastique, 21 sept. 1794, 30 juin 1794, Joseph, Marguerite Trahan.

Hébert, Scholastique, 5 mai 1776, -, Jean-Charles, Magdeleine Robichaud.

Henry, Jean-Baptiste, 10 juin 1779, 18 août 1778, Jean, Marguerite Nope.

Judice, Godefroy, 6 janv. 1779, 11 avril 1778, Louis, ${ }^{10}$ Marguerite Patin.

Judice, Marie-Zéline, 21 sept. 1794, 20 août 1793, Louis, Marguerite Patin.

Labauve, Anne, 25 avril 1773, 28 sept. 1772, Jean-Baptiste, Françoise Broussard.

Labauve, François, 5 mai 1776, -, Jean-Baptiste, Françoise Broussard.

Labauve, Jean, 25 avril 1771, 8 avril 1771, Jean-Baptiste, Françoise Broussard.

Labbé, Célestin, 10 janv. 1779, 7 sept. 1778, Jean, M.-Françoise Bara.

Labbé, Dorothée, 10 janv. 1779, 7 sept. 1778, Jean, M.-Françoise Bara.

Labbé, Euphrosine, 24 nov. 1776, 12 janv. 1775, Jean, M.-Françoise Bara.

Labbé, Jean-Baptiste, 10 janv. 1779, 7 sept. 1778, Jean, M.-Francoise Bara.

Landry, Françoise, 2 janv. 1771, 22 oct. ....., Firmin, Théotiste Thibodeau.

Landry, Hélène, 1774, 20 nov. 1774, Firmin, Théotiste Thibodeau.

Landry, Hubert, 29 avril 1773, le jour préc., Firmin, Théotiste Thibodeau.

Landry, Isidore, 27 juill. 1765, la veille, Mathurin, Marie Dugas. 10 Louis Godefroy, lieutenant de milice. 
Landry, Rosalie, 24 nov. 1776, 15 août 1776, Firmin, Théotiste Thibodeau.

Landry, Victoire, 5 mai 1776, le même jour, Amand, Marguerite Melançon.

Leblanc, Agricole, 29 avril 1773, 13 nov. 1772, Simon, Marguerite Guilbaut.

Leblanc, Céleste, 22 août 1777, 7 mars 1777, René, Marguerite Trahan.

Leblanc, Frédéric, 2 janv. 1771, 3 fév. 1770, Simon, Marguerite Guilbaut.

Leblanc, Jean-Baptiste, 5 mai 1776, -, Pierre, Anastasie Louvière.

Leblanc, Joseph, 1 déc. 1776, 14 nov. 1776, Simon, Marguerite Guilbaut.

Leblanc, Marguerite, 9 sept. 1774, 9 sept. 1774, Simon, Marguerite Guilbaut.

Leblanc, Pierre, 19 juill. 1778, 29 juin 1778 ou 1776, Simon, Marguerite Guilbaut.

Martin, Apollinie, 5 mai 1776, _, Claude, Marie Bonin.

Martin, Françoise-Pélagie, 25 av. 1773, 20 janv. 1773, Joseph, Isabelle Thibodeau.

Martin, Jean-André, 2 janv. 1771, 1 sept. 1770, Claude, Marie Babin.

Martin, Joseph, 13 avril 1773, 27 janv. 1773, Claude, Marie Babin. Martin, Michel, ${ }^{4 b} 25$ août 1777, 6 mars 1777, Claude, Marie Babin. Meaux, Antoine, 5 mai 1776, - , Michel, Elisabeth Broussard.

Meaux, Céleste, 18 juill. 1772, 30 juill. 1771, Michel, Isabelle Broussard.

Meaux, François-Xavier, 26 août 1777, 23 avril 1777, Michel, Elisabeth Broussard.

Meaux, Michel, 5 avril 1773, 2 fév. 1773, Michel, Isabelle Broussard.

Nezat, M.Françoise, 19 juill. 1778, 6 mars 1778, Pierre, Magdeleine Provost.

Nézat, Marie-Julie, 29 avril 1773, 15 déc. 1772, Pierre, Magdeleine Provost.

Patein, Marguerite, 8 janv. 1779, 15 nov. 1777, Antoine, Catherine Beaussier.

Pèlerin, Eugénie, 17 avril 1773, 8 mars 1772, Grégoire, Cécile Préjean.

Pèlerin, Frédéric, 17 avril 1773, 10 déc. 1770, Grégoire, Cécile Préjean.

Pèlerin, Marie, 11 janv. 1766, âgée de 4 mois, Charles, Isabelle Thibodeau. 
Provost, Félicité, 15 juill. 1772, 1 fév. 1772, Nichols, M-Françoise Thibodeau.

Provost, Julie, 15 juill. 1778, 13 mars 1775, François, Geneviève Benoit.

Provost, Marie, 15 juill. 1778, 3 mai 1777, François, Geneviève Benoit.

Savoy, M-Céleste, ${ }^{4 b} 25$ août 1777, 14 août 1777, François, Marie Martin.

Semer, Jean-Baptiste, 24 nov. 1776, 3 sept. 1776, Jean-Baptiste, Marie Thibodeau.

Semer, Victoire, 1774, 27 avril 1774, Jean-Baptiste, Marie Thibodeau.

Sémaire, Urbain, 17 juill. 1772, 22 juill. 1771, Jean-Baptiste, Marie Thibodeau.

Simar, Louis, ${ }^{11}$ - , janv. 1793, Louis, Marie Trahan.

Simar, Marie, ${ }^{11}$ - , janv. 1791, Louis, Marie Trahan.

Soudric, Martin, 29 avril 1773, 21 avril 1773, Martin, Perrine Bara.

Suret, Augustin, 19 juin 1765, le même jour, Pierre, Marie Thibodeau.

Thibodeau, André Paul, 27 août 1765, la veille, Paul, Rozalie Guilbaut.

Thibodeau, Cyrille, 1774, 8 oct. 1773, Olivier, Agnès Brun.

Thibodeau, Elizabeth, 4 nov. 1776, 4 sept. 1776, Paul, Rosalie Guilbaut.

Thibodeau, Gertrude, -, 30 janv. 1778, Aman, Gertrude Bourque.

Thibodeau, Jean-Baptiste, 1774, 24 nov. 1774, Aman, Gertrude Bourque.

Thibodeau, Joseph, 19 juill. 1778, 4 janv. 1778, Paul, Rosalie Guilbaut.

Thibodeau, Louise, 17 juill. 1772, 22 sept 1771, Aman, Gertrude Bourque.

Thibodeau, Marguerite-Anne, 11 mai 1765, 16 mai 1765, Olivier, Magdeleine Broussard.

Thibodeau, Nicolas, 17 juill. 1772, 15 1771, Olivier, Agnès Brun. Thibodeau, Olivier, 15 mai 1776, -, Olivier, Agnès Brun.

Thibodeau, Séraphie, 2 janv. 1771, 15 oct. 1770, Paul, Rozalie Guilbaut.

Thibodeau, Vital, 29 avril 1773, 9 oct. 1772, Paul, Rosalie Guilbaut.

\footnotetext{
11 Les certificats de naissance de ces deux enfants ont été trouvés sur des coupons qui ont été faits par le Père Bernard de Deva, Capucin, curé de cette paroisse et ne portent aucune signature ni date de baptême (note du curé Migl Bernd Dassiese, curé de St-Martin des Atakapas, qui a collectionné tous les actes de ce registre).
} 
Trahan, Julienne, 19 juill. 1778, 1 nov. 1777, Paul, Marie Dugas Duhon?

Trahan, Louis-Joseph, 25 avril 1773, 19 août 1772, René, Isabelle Broussard.

Trahan, M-Magdeleine, 6 janv. 1779, 24 juin 1778, Athanase, Magdeleine Thibodeau.

Trahan, Paul, 5 mai 1776, -, Paul, Marie Duhon.

Trahan, Reine, 1774, 29 janv. 1773, Paul, Marie Duhon.

Wilse, M-Marguerite, 22 oct. 1774, le même jour, Philippe, Marie Roze Dorat.

Wilse, Philippe, 25 août 1777, 5 août 1776, Philippe, Marie Dausa.

?, Marie-Louise, 18 juill. 1778, —, ?, Jeanne Provost.

?, Zénon, ${ }^{1} 26$ août 1777, 10 janv. 1777, ?, Magdeleine.

Hector appelé Philippe, 30 ans environ, esclave de M. Bérard.

\section{MARIAGES}

Bonin, Antoine, natif de la Mobile, f. d'Antoine et de Marie Hellier, m. le 11 janv. 1779 avec Magdeleine Prévost, native de la Pointe Coupée.

Bonin, Jean-Louis, natif des Alibamons, f. d'Antoine et de Marie Tellier, m. le 25 avril 1771, avec Marguerite Prince, native de l'Acadie, f. d'Olivier et de Marguerite Boudreau.

Boutte, les deux frères mariés aux deux sœurs Degruis, le 12 juillet 1778.

Broussard, René, veuf de Magdeleine Landry, natif de l'Acadie, f. de Firmin et de Françoise Thibodeau, m. le 19 janvier 1779, avec Anne Gaudin, f. de Bonaventure et de Théotiste Thibodeau.

Cormier, Jean-Baptiste, veuf de Marguerite Bourque, de la paroisse des Opelousas, m. le 10 janvier 1779, avec Anne Blanchard, veuve de Joseph Richard de la paroisse des Opelousas.

Descuir, Francois, natif de la Pointe Coupée, f. de feu Jean et de Geneviève Moieux, $\mathrm{m}$. le 1er mai 1773 , avec Marie Labbé, native de Pointe Coupée, f. de Jean et de Jeanne Julie Ozenne.

Doucette, Joseph, f. de Michel et de Marguerite Martin, m. le 18 juillet 1772, avec Anne. Landry, f. de Jean et de Magdeleine Broussard.

Dugas, Aman, natif de l'Acadie, habitant Saint-Joseph des Atakapas, f. de Claude et de Anne Hébert, $m$. le 11 janvier 1779, avec Geneviève Robichaud f. de René et de Marguerite Martin. 
Dugas, Pierre, f. de Charles et de Anne Robichaud, m. le 18 juillet 1772, avec Anne Thibodeau, f. de Charles et de Brigitte Breau. (3e degré d'affinité).

Fostin, Jacques, natif des Illinois, f. de Jacques et de Françoise Eren, m. le 18 juillet 1772, avec Françoise Trahan f. de Michel et d'Euphrasie Vincent.

Fuselier de la Clair, Gabriel, Commandant pour le Roy et juge au quartier des Atakapas et Opelousas, natif de Lyon, paroisse Saint Nisier, veuf de Jeanne Roman f. de feu Pierre, Capitaine de milice et de Ludivine Chanfouran, m. le 30 avril 1771, avec Hélène Soileau, native des Natchez, f. de feu Noel, garde magasin pour le Roy au poste des Natchez, et de Marie Josephe Richanine.

Granger, Jean-Baptiste, natif de l'Acadie, paroissien des Opelousas, f. de Pierre et d'Euphrosine Gaudreau, m. le 10 janvier 1779, avec Suzanne Cormier, f. de Joseph et de Marguerite Sonnié, de la même paroisse.

Guilbaut, François, f. de Joseph, m. le 18 juillet 1772, avec Magdeleine Broussard, f. de Jean et de Anne Leblanc.

Hébert, Charles, natif de l'Acadie, f. de Bénonie et d'Anne Savoy, m. le 27 avril 1773 avec Magdeleine Robichaud, native de l'Acadie, f. de feu René et de Marguerite Martin.

Richard, Fabien, f. de Pierre et de Marguerite Dugas, m. le 10 janvier 1779, avec Françoise Thibodeau, f. de Pierre et de Françoise Sonier.

Sonier, Joseph, f. d'Etienne et de Anne des Bois, de la paroisse des Opelousas, $m$. aux Opelousas, le 10 janvier 1779 , avec Marie Thibodeau, f. d'Olivier et de Magdeleine Broussard.

Trahan, Paul, f. de Michel et d'Euphrasie Vincent, m. le 18 juillet 1772, avec Marie Duhon, f. de Joseph et de Théotiste Broussard.

\section{SEPULTURES}

Certains certificats de sépulture mentionnent la date d'entrée au registre et la date et le lieu d'inhumation, aux Atakapas.

Arsenault, François, 22 sept. 1765, a été enterré au premier Camp d'en bas le 19.

Bellefontaine, Joseph, 6 sept. 1765, a été enterré au premier Camp d'en bas le 2 sept.

Bergeron, Augustin, 31 août, a été enterré hier au premier Camp d'en bas.

Bergeron, Barthélémy, 30 oct. 1765, a été enterré au premier Camp d'en bas.

Bergeron, Charles (environ huit mois) 30 oct 1765, a été enterré le 29, au premier Camp d'en bas. 
Bergeron, Jean-Baptiste, 23 nov. 1765, a été enterré le 2, au premier Camp d'en bas.

Bergeron, Joseph, ? oct. 1765 (15 mois).

Bernard, Michel (9 mois), 30 oct. 1765, a été enterré le 28 au premier Camp.

Breau, Brigitte, 5 août 1765 , veuve de feu Charles Thibodeau.

Breau, Sylvain, 12 oct. 1765, enterré au dernier Camp d'en bas.

Broussard, Alexandre, 22 sept. 1765, enterré le 18, au Camp d'en bas.

Broussard, Joseph, 7 sept. 1765, enterré le 5 au dernier Camp d'en bas.

Broussard, Magdeleine, 17 mai 1765, décédée la veille, épouse d'Olivier Thibodeau.

Broussard, Théotiste, 27 juillet 1765, enterré le 26, au dernier Camp d'en bas (n'ayant pu être emporté à cause de l'éloignement).

Darcy, Isabelle, 12 oct. 1765, enterrée le 10 au dernier Camp d'en bas.

Doucet, Marie-Marthe (20 mois), 24 nov. 1765.

Dugas, Jean, 6 sept. 1765, enterré le 5 au premier Camp d'en bas.

Dugas, Jean, 22 sept. 1765, enterré le 19, au premier Camp d'en bas.

Dugas, Joseph (habitant), 27 juillet 1765, décédé la veille.

Dugas, Joseph, 8 oct. 1765, au premier Camp d'en bas.

Dugas, Magdeleine, 6 oct. 1765 .

Dugas, Marie, 9 juillet 1765, enterrée au premier Camp d'en bas, épouse de Mathurin Landry.

Girouard, Joseph, 3 nov. 1765, enterré le 22 oct. au premier Camp d'en bas.

Godin, Marie-Charlotte, 18 juillet 1765, décédée la veille, épouse de Jean Dugas, habitant.

Guilbaut, Joseph, officier, 1er sept. 1765, décédé la veille.

Hébert, Pierre, orphelin de père et de mère, 25 juillet 1765, décédé la veille.

Hugon, Jacques, 12 oct. 1765, enterré le 8 au Camp d'en bas.

Joseet, Paul, 24 août 1765, décédé ce jour.

Lagresse, ?, 1er août 1765 .

Leblanc, Isabelle, 29 oct. 1765, épouse de Victor Broussard.

Pelletier de la Houssaye, chevalier de l'Ordre de Saint-Louis, ancien major de la Place de la Nouvelle-Orléans, époux de Victoire Petit Delevelier, sép. paroisse Saint-Joseph des Atakapas, le 22 nov. 1777.

Petit de Levilier, Antoinette Pélagie, 26 avril 1773, décédée le 7 déc. 1772, épouse de Vaugine de Nuisement, capitaine des armées de Sa Majesté Catholique. 
Poirier, Jean Chrysostome, 1er juillet 1765 (18 mois). Robichaud, Marin, 18 août 1765, décédé ce jour.

Robichaud, René, 2 août 1765, décédé la veille.

Thibodeau, Catherine, 15 nov. 1765 , épouse de Simon Leblanc.

Thibodeau, Marguerite, 7 sept. 1765, enterrée le 5, au dernier Camp d'en bas, épouse d'Alexandre Broussard.

Thibodeau, Marguerite-Anne, 16 mai 1765, f. d'Olivier et de Magdeleine Broussard.

Trahan, Visule, 12 oct. 1765, enterré le 10 au dernier Camp d'en bas.

(à suivre)

JEANNE GRÉGOIRE 